\title{
PERFEZIONAMENTI APPORTATI AD UN APPARATO FAC-SIMILE
}

\author{
Marco Frank
}

In un precedente articolo ${ }^{1}$ ) veniva descritto un apparato facsimile per la riproduzione di carte meteorologiche. La caratteristica principale di questa apparecchiatura era rappresentata dal fatto che la sotloportante forniva al ricevitore sia l'informazione relativa all'immagine, mediante la sua modulazione d'ampiezza, sia l'informazione concernente la velocità di rivoluzione del cilindro su cui è avvolto il documento da trasmettere. Infatti nell'analizzatore vi era un oscillatore che produceva una frequenza di $300 \mathrm{c} / \mathrm{s}$. La tensione a $300 \mathrm{c} / \mathrm{s}$ alimentava, attraverso adatti amplificatori di potenza, sia il motore sincrono del cilindro, sia un motore sincrono collegato ad un disco recante una serie di fori o di tagli presso il bordo. Il disco, interposto tra la sorgente luminosa e l'immagine da trasmettere, girando intercettara periodicamente il raggio di luce. La luce intermittente, diffusa dal punto esplorato, era raccolta da una fotocellula la cui uscita costituiva direttamente la sottoportante modulata; la frequenza della sottoportante era pertanto collegata alla velocità di rotazione del cilindro.

Nel registratore la sottoportante, dopo una prima amplificazione, era inviata sia ad un demodulatore e ad un complesso elettromeccanico scrivente, sia ad un limitatore che sopprimeva le fluttuazioni dovute alla modulazione. La tensione rettangolarizzata dal limitatore sincronizzava una serie di divisori a multivibratore che dividerano la frequenza della sottoportante fino a riottenere i $300 \mathrm{c} / \mathrm{s}$, con i quali veniva alimentato un motore sincrono che faceva girare il cilindro sul quale avveniva la registrazione.

Questa apparecchiatura, nonostante i suoi numerosi difetti, consenti un collegamento giornaliero per 60 giorni su 60 di prova, su una linea telefonica di una quindicina $\mathrm{di} \mathrm{km}$.

I principali difetti inerenti al sistema di sincronismo erano $\mathbf{i}$ seguenti :

(1) Annali di geofisica, vol. II, n. 4, 19.19, pagg. 532-544. 


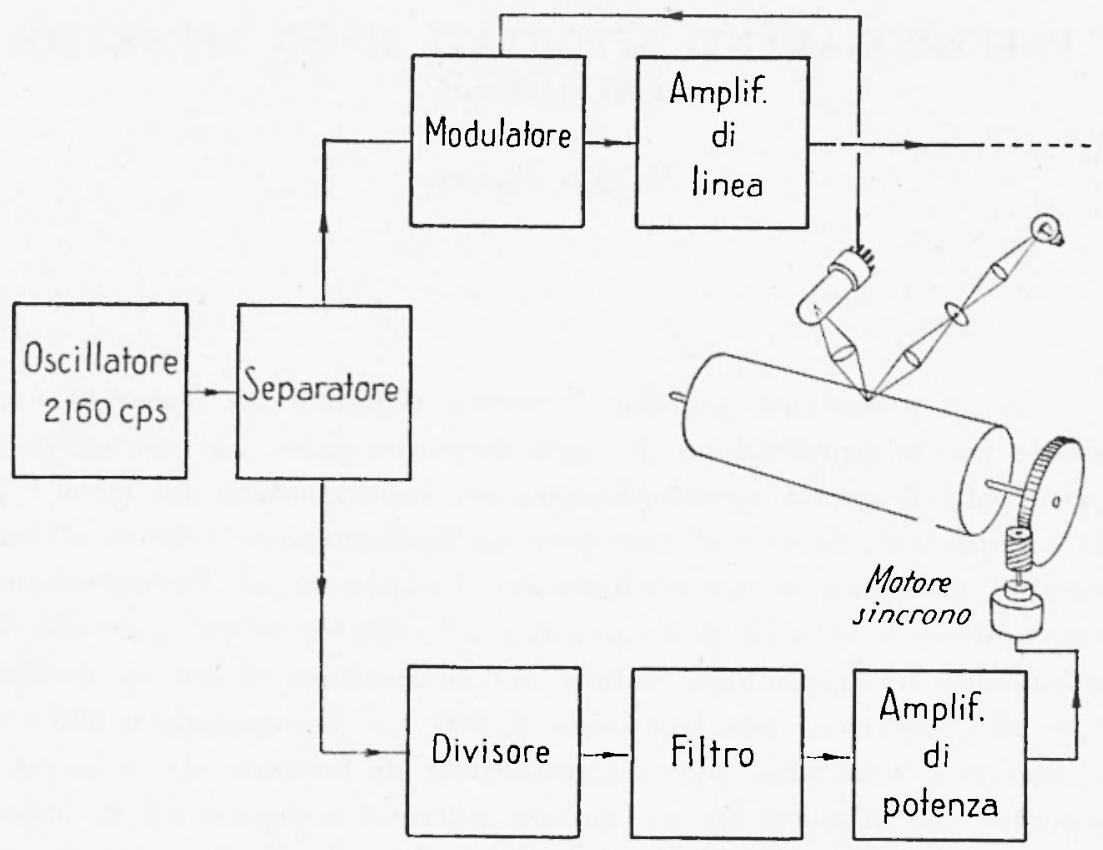

Fig. 1

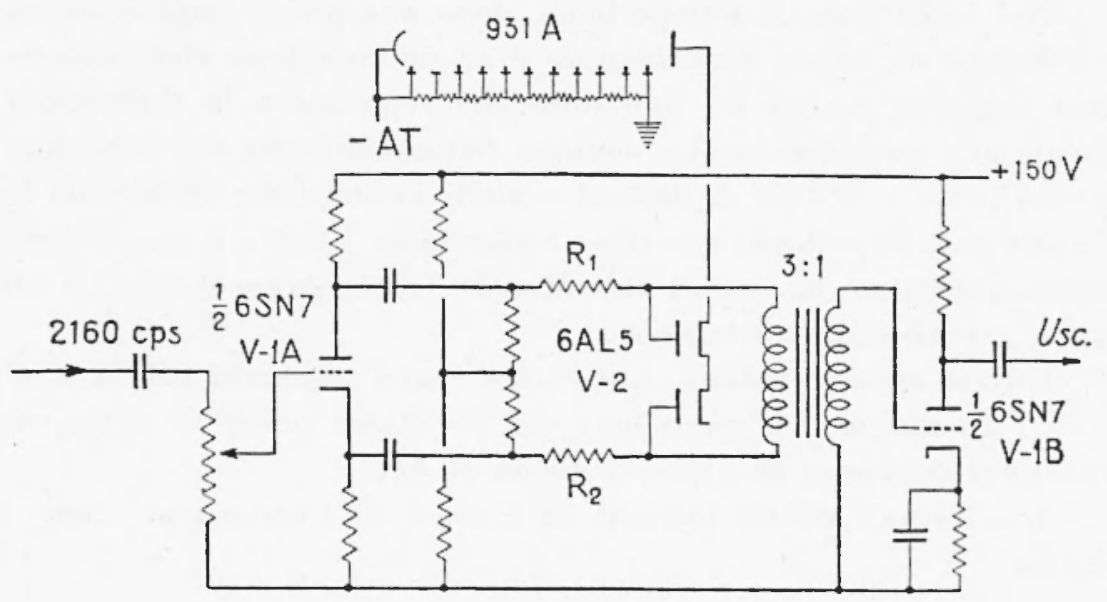

Fig. 2 
1) Non è possibile effettuare una modulazione d'ampiezza senza introdurre contemporaneamente una modulazione di fase, per cui la divisione della frequenza effettuata dai multivibratori si fa tanto più critica quanto più alta è la percentuale di modulazione. La divisione fu effettuata anche con circuiti contatori, ma comunque le fluttuazioni di fase, anche se piccole, influivano sul motore sincrono riducendo la qualita dell'immagine registrata.

2) Una linea di trasmissione, quale un cavo telefonico, introduce

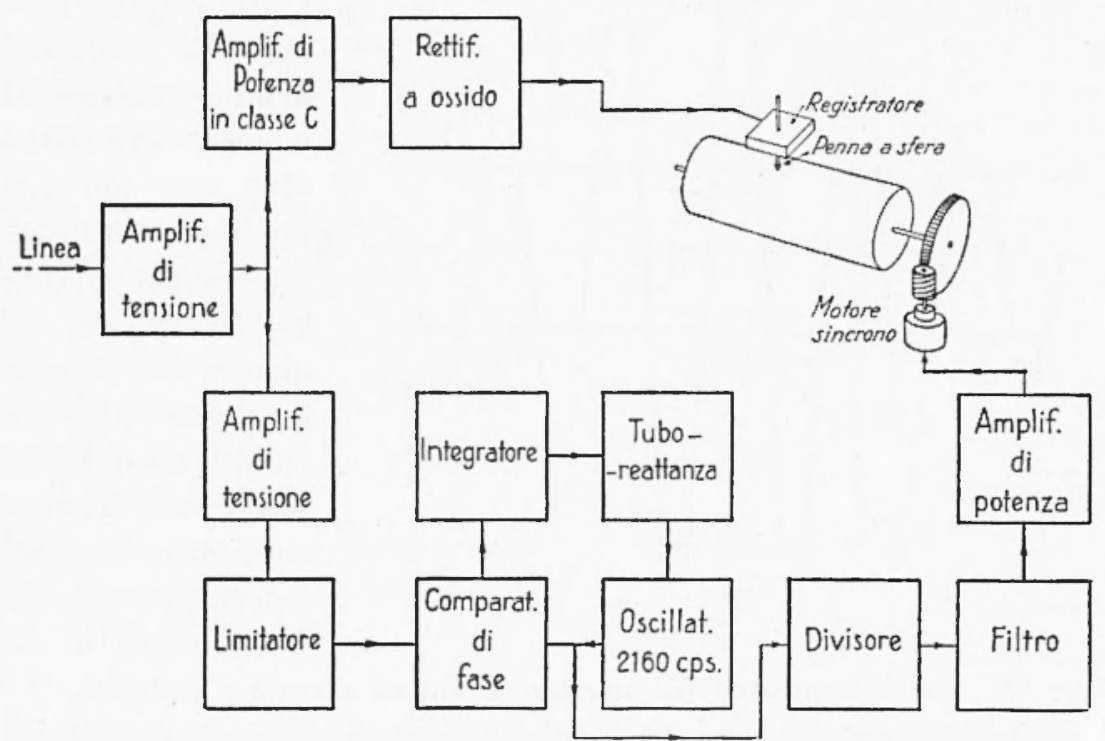

Fig. 3

una distorsione di fase che comporta lo stesso inconveniente sopra lamentato.

3) La trasmissione via radio di un'immagine era impossibile in quanto per ogni disturbo o interruzione, anche brevissima, il divisore ed il motore sincrono perdevano la fase, e la registrazione si arrestava.

D'altra parte l'assoluta assenza di distorsione nella registrazione, la possibilità di costruire l'apparecchiatura con materiale corrente, ed il continuo progresso nei collegamenti consigliarono di sviluppare questo sistema di sincronismo, per eliminarne i principali difetti, senza per questo abbandonare definitivamente il sistema di sincroni- 
smo mediante diapason, al quale spettano prerogative notevolissime, a cui si accennerà più avanti in questo articolo.

In base alle considerazioni esposte, si sono ridisegnati i circuiti, pervenendo alle attuati apparecchiature.

Analizzatore. - Lo schema a blocchi dell'analizzatore è rappresentato in fig. 1 .

In questa apparecchiatura si è preferito partire dalla frequenza

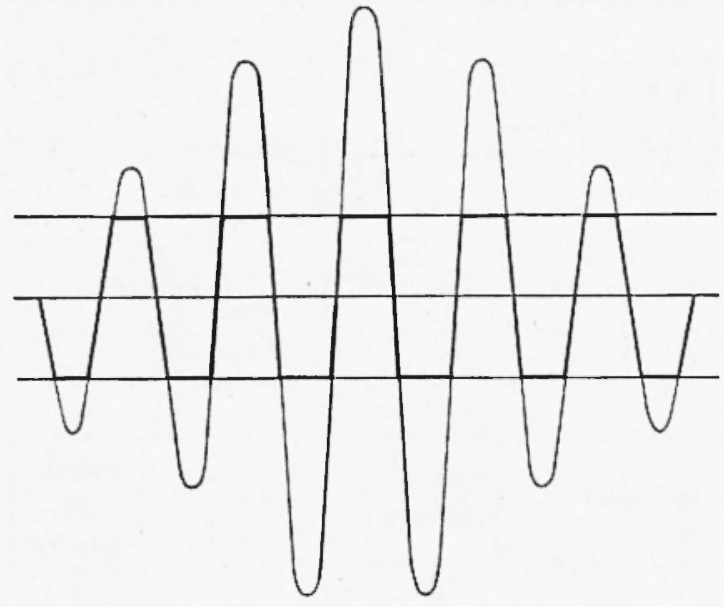

Fig. 4 della sottoportante $(2160 \mathrm{c} / \mathrm{s})$, ed ottenere la frequenza di alimentazione del motore sincrono $(360 \mathrm{c} / \mathrm{s})$ con una divisione elettronica, anziché partire dalla frequenza del motore ed ottenere la sopportante con un sistema di moltiplicazione elettromeccanico come nel sistema precedente. L'oscillatore ì del

tipo $R C$, a sfasamento ed ha una buona forma d'onda e stabilità.

E da notare che in questo sistema di sincronismo la stabilità di frequenza di questo oscillatore non la particolare importanza, in quanto una variazione relativa della frequenza influenza nella stessa misura percentuale sia la velocità di rivoluzione del cilindro dell'analizzatore che quella del cilindro del registratore.

La frequenza di $2160 \mathrm{c} / \mathrm{s}$ è stata scelta come segue: si è partiti dalla frequenza di $2700 \mathrm{c} / \mathrm{s}$, considerabile come la frequenza di taglio di molte linee telefoniche, si è sottratta la banda di frequenze corrispondente ai segnali dell'immagine, e si è ottenuto così un valore di circa $2100 \mathrm{c} / \mathrm{s}$.

Si è scelta allora la frequenza di $2160 \mathrm{c} / \mathrm{s}$, prossima al valore di 2100 , che rappresenta il sesto multiplo di $360 \mathrm{c} / \mathrm{s}$, frequenza adatta ad alimentare il motore sincrono, dato il numero di poli di questo, il rapporto di trasmissione tra motore e cilindro, e la velocità di rivoluzione richiesta per quest'ultimo (1 giro sec.). 
Essendosi tenuta la frequenza della sottoportante nella parte più alta della gamma delle frequenze telefoniche, resta una zona inferiore utilizzabile per telescriventi o per una comunicazione telefonica di cattiva qualità, ma ancora comprensibile.

La tensione prodotta dall'oscillatore va ad un doppio triodo che funziona da separatore per impedire che impulsi a $360 \mathrm{c} / \mathrm{s}$ prodotti dal divisore possano entrare nella modulazione. L'uscita di uno dei triodi va al modulatore, l'altra va al divisore. Lo schema del modulatore ̀̀ rappresentato in fig. 2.

Il triodo $V-1 A$ ( $1 / 2$ 6SN7) serve a passare da una tensione monofase a c/s ad una tensione in controfase, necessaria al modulatore. In questo ì in:piegato un doppio diodo $V$-2A e $B$

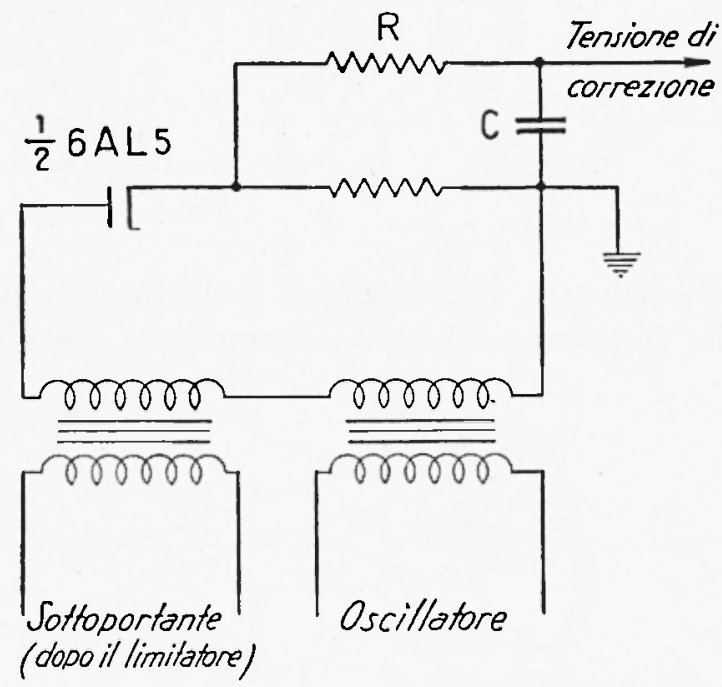

Fig. 5 (6AL5).

Quando il fotomoltiplicatore (931 $A$ ) non è illuminato, la placca di questo si trova circa al potenzione di cresta (positivo) delle placche del doppio diodo e non assorbe sensibile corrente. Pertanto nelle resistenze $X$ di placca dei due diodi ( $R-1$ ed $R-2)$ non si ha altra caduta che quella (trascurabile) dovita al carico del primario del trasformatore. Viceversa quando il fototubo è illuminato, tra la placca e l'ultimo dinodo si ha una resistenza o piuttosto bassa. Considerando per approssimazione sia infinita, e che l'indipendenza del primario del trasformatore, detta $E$ la tensione tra placca e catodo del triodo $V \cdot 1 A$, la tensione ai capi del primario è quindi ancora $E$ quando il fototubo non $\grave{c}$ illuminato, e si riduce ad $E_{\varrho} /(R+. \varrho)$ quando il fototubo è illuminato: il rapporto $\varrho /(R+\varrho)$ risulta di circa 0,15 quando è esplorato un punto bianco dell'immagine. Con questo medulatore si realizza pertanto una profondità massima di modulazione dell' $85 \%$ circa.

La sottoportante modulata è successivamente amplificata dal trio- 
do $V-1 B(1 / 26 S N 7)$ e dal tetrodo $V .3$ (6AQ5). L'uscita di questo è inviata sulla linea.

La tensione a $2160 \mathrm{c} / \mathrm{s}$ prodotta dall'oscillatore, attraverso il trio-

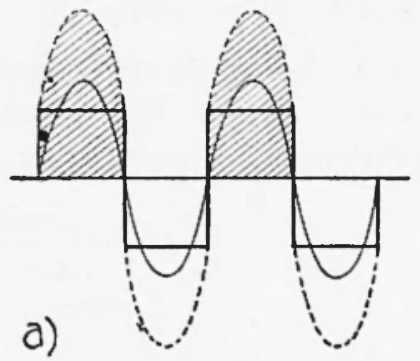

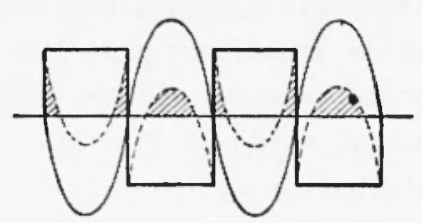

b)

Fig. 6

do separatore, sincronizza un multivibratore il quale fornisce impulsi alla frequenza del sesto sottomultiplo della frequenza di $2160 \mathrm{c} / \mathrm{s}$,

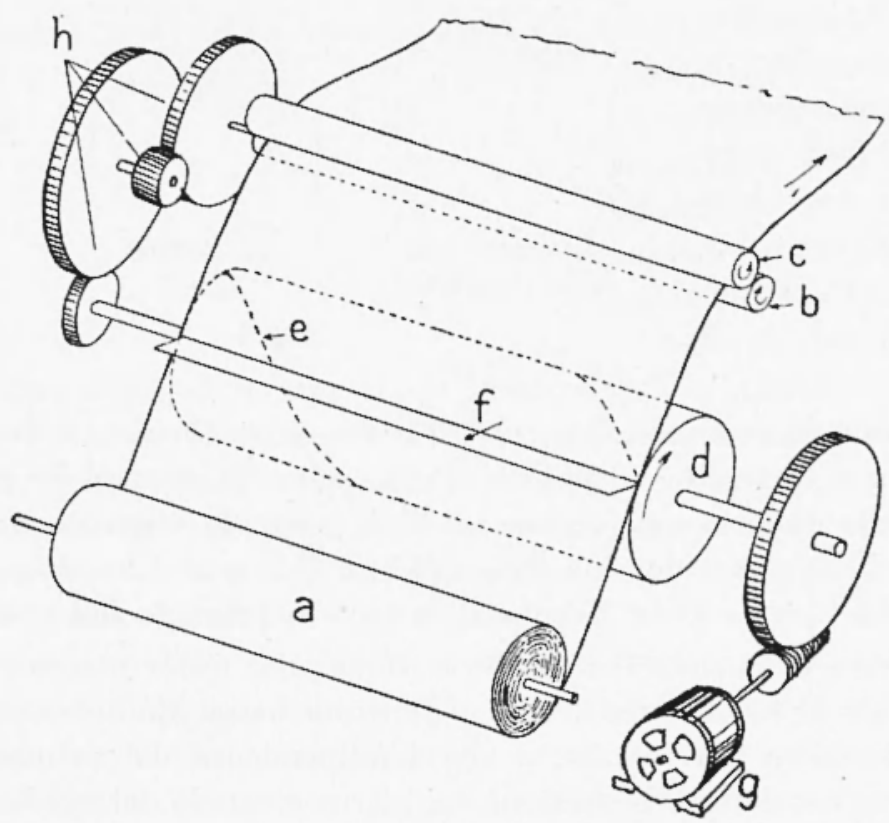

Fig. 7

ossia a $360 \mathrm{c} / \mathrm{s}$. Quest'ultima frequenza è filtrata ed inviata ad un amplificatore di potenza la cui uscita alimenta il motore sincrono.

Questo, che ha dieci coppie polari, fa 36 giri/sec.

Il cilindro sul quale è avvolto il documento da trasmettere, è 
comandato da un motore con un rapporto $36 / 1$, per cui compie una rivoluzione al secondo.

Un tubo a raggi catodici, alimentato dalla stessa alta tensione che alimenta il fotomoltiplicatore, permette un controllo della divisione mediante una figura di Lissajous ottenuta applicando come asse $X$ una tensione a $360 \mathrm{c} / \mathrm{s}$, e come asse $Y$ una tensione a $2160 \mathrm{c} / \mathrm{s}$. Con un commutatore è possibile portare come asse $X$ una tensione a

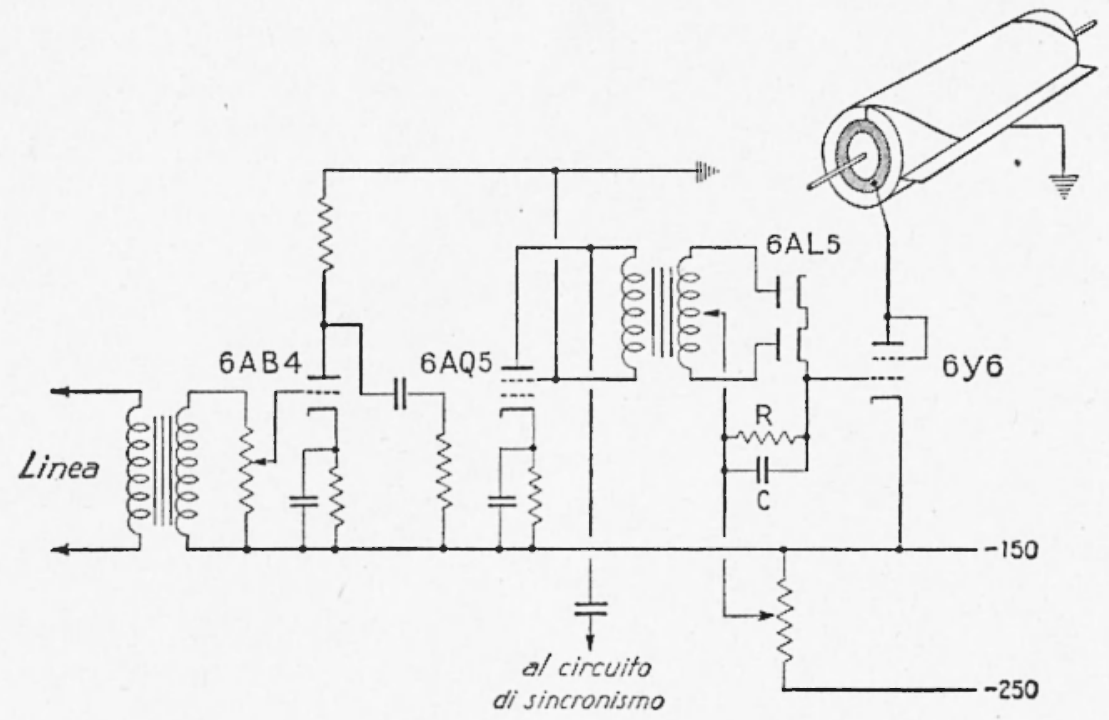

Fig. 8

frequenza di rete, e come asse $Y$ la sottoportante modulata, ed ottenere così un controllo della morlulazione inviata sulla linea.

Registratore. - Lo schema a blocchi del registratore è rappresentato in fig. 3. La sottoportante modulata è amplificata e successivamente viene utilizzata sia per la registrazione, sia per il sincronismo. Come si è visto, la morlulazione è negativa nel senso televisivo, ciò̀ il massimo di ampiezza corrisponde al nero, ed il minimo al bianco. Pertanto in un amplificatore di potenza, opportunamente polarizzato, al bianco corrisponde un'uscita nulla, ed al nero un'uscita massima.

L'uscita di questo amplificatore, costituito da due $6 L 6$ in controfase è raddrizzata mediante un rettificatore ad ossido per le due semionde, ed applicata alla bobina mobile di un altoparlante magnetodinamico che porta la penna a sfera in luogo della membrana. 


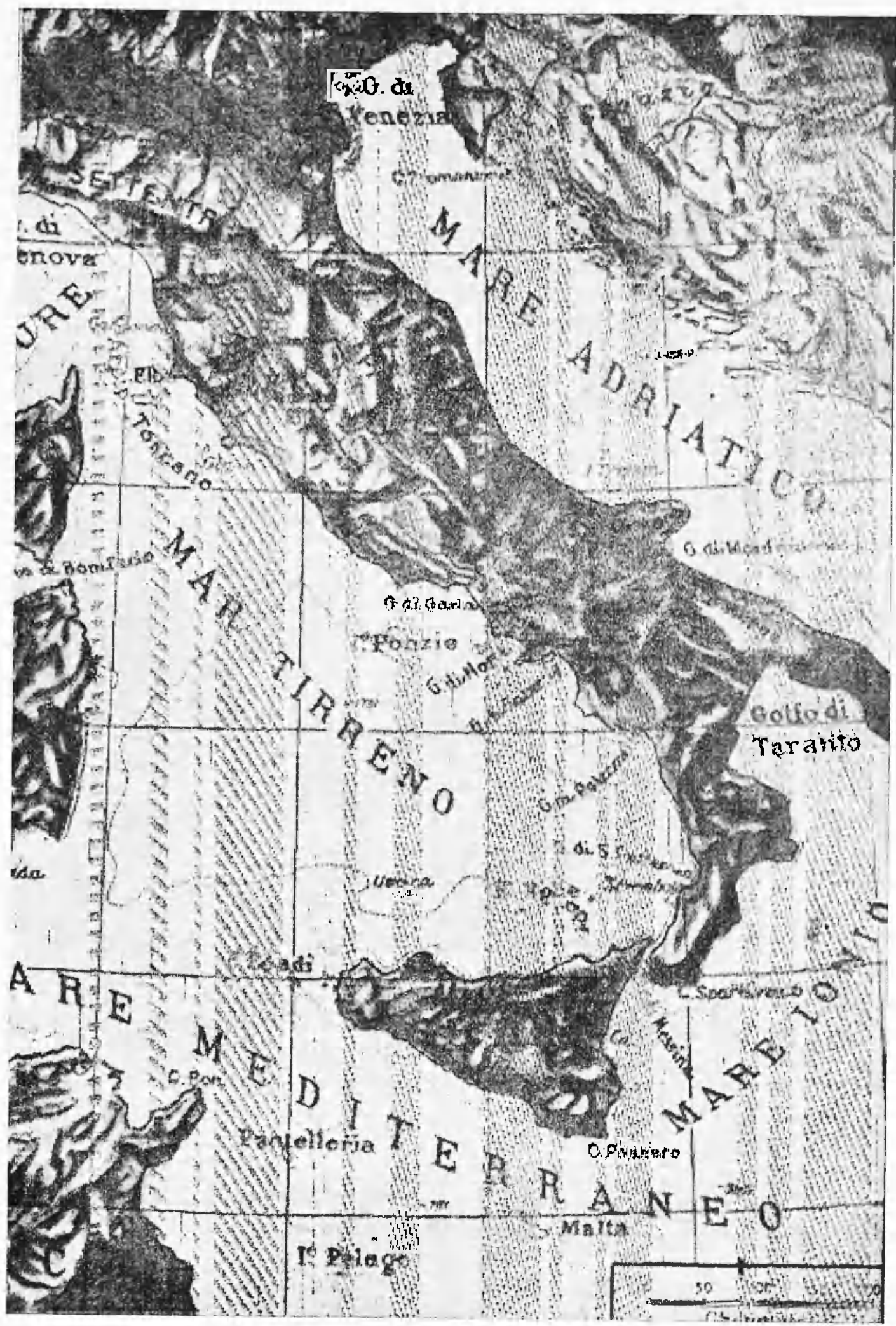

Fig. 9 
La penna sfiora il cilindro su cui c avolta la carta per la registrazione. Come nel tipo precedente, oltre ad una prima copia a penna si può avere un certo numero di copie con carta carbone, oppure

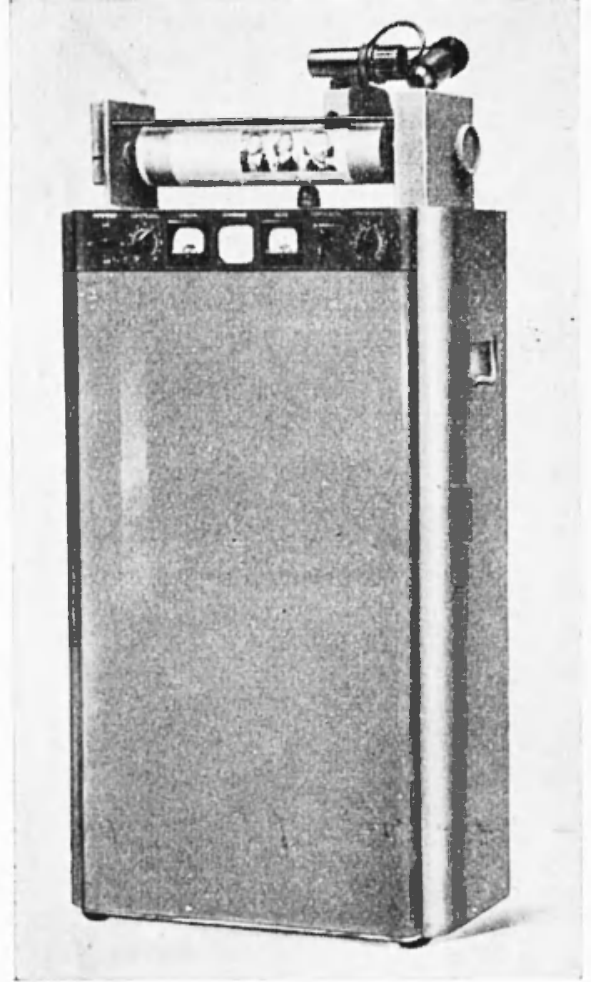

Fig. 10

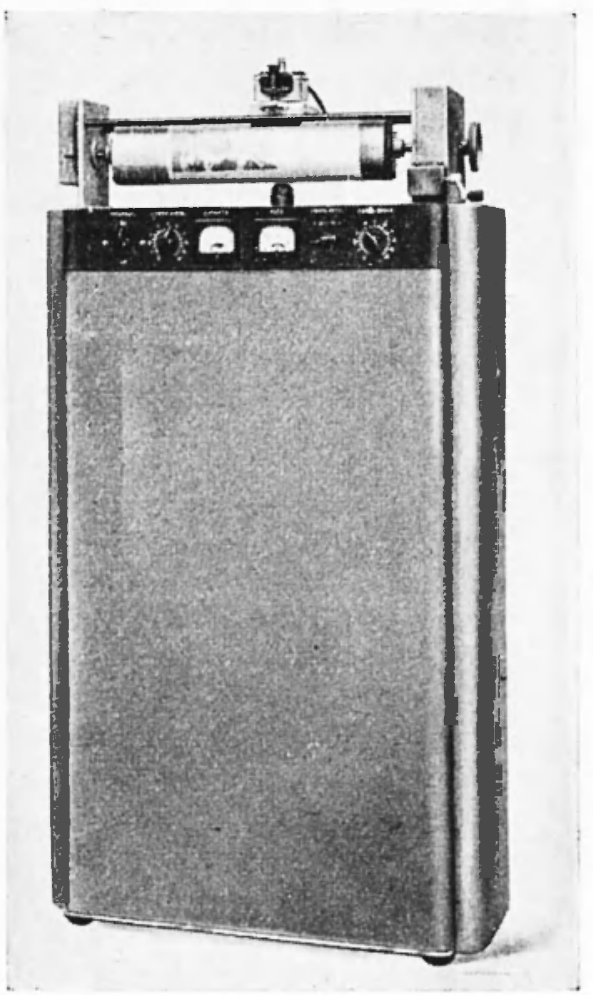

Fig. 11

una matrice ottenuta con carta carbone copiativa, per ciclostile ad alcool.

Per quanto riguarda il sincronismo, la sottoportante, dopo essere stata ulteriormente amplificata, è inviata ad un limitatore che ne lascia passare solo una ristretta porzione centrale (fig. 4).

La tensione cosi ottenuta va al comparatore di fase, il cui schema è rappresentato in fig. 5. A questo comparatore, oltre alla tensione rettangolare della sottoportante, è applicata l'uscita sinusoidale di un oscillatore.

La fig. 6.4 mostra l'oscillogramma della tensione applicata alla placca del diodo rettificatore (linea punteggiata) quando le due ten- 
sioni corrispondenti alla sottoportante ed all'oscillatore sono in fase.

La tensione all'uscita dell'integratore $R C$ della fig. 5 è proporzionale all'area tratteggiata, costituendone il valor medio.

Quando le due tensioni sono in opposizione di fase (fig. $6 \mathrm{~B}$ ), l'area è molto minore, e l'uscita dell'integratore è altrettanto ridotta.

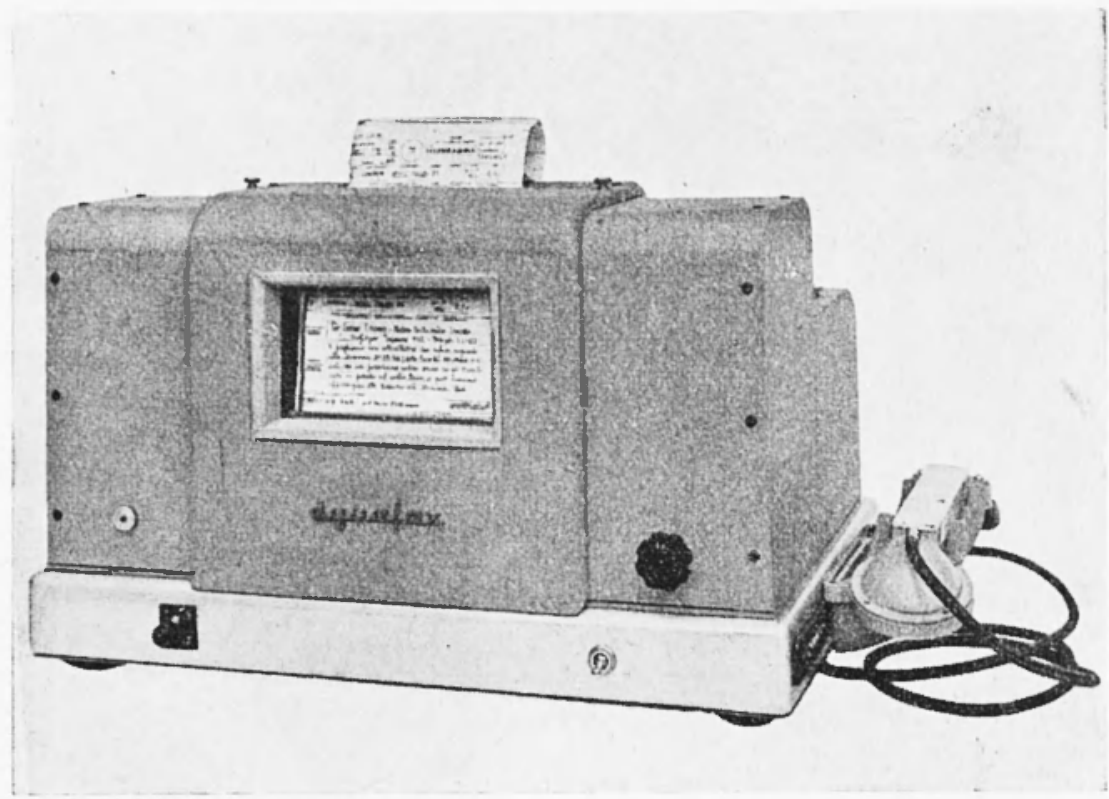

Fig. 12

A condizioni intermedie della relazione di fase corrispondono uscite intermedie.

In parallelo al circuito oscillante dell'oscillatore è connesso un tubo reattanza $(6 L i)$, a cui è applicata la tensione di correzione fornita dal comparatore di fase. La tensione dell'oscillatore è in ritardo rispetto alla tensione della sottoportante. Se la frequenza deli'oscillatore tende ad aumentare rispetto alla frequenza della sottoportante, il ritardo diminuisce, la tensione di correzione ai capi di $C$, prodotta dal comparatore cresce, la reattanza (capacitiva) del tubo cresce e la frequenza si mantiene cosi allo stesso valore della frequenza della sottoportante. Analogo processo (inverso) avviene se la frequenza dell'oscillatore tende a diminuire rispetto alla frequenza della sottoportante.

È da notare che questo sistema è largamente usato in ognì caso 
in cui sia necessario il controllo automatico di una frequenza: ma la particolarità di questa applicazione risiede in ciò: la costante di tempo $R C$ ì dimensionata in modo tale non solo da essere grande rispetto al periodo, ma laa il valore più grande possibile (circa 0,1 sec.) compatibilmente con la necessità che la tensione di correzione possa seguire le fluttuazioni relative tra le frequenze prodotte dai due oscillatori, rispettivamente posti nell'analizzatore e nel registratore.

Infatti l'integratore, oltre a completare il circuito del comparatore di fase ed a costituire il necessario filtro passa-basso, ha l'importante funzione di "parastrappi " e di "volano", cioc assorbe eventuali brevi interruzioni e disturbi della sottoportante, mantenendo costante la relazione tra la fase dell'oscillatore del registratore e la fase media della sottoportante.

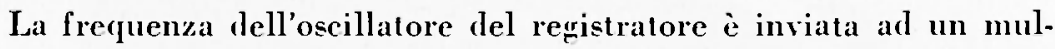
tivibratore il quale divide la frequenza suddetta per 6 , come nell'analizzatore, ottenendosi cosi la frequenza di $360 \mathrm{c} / \mathrm{s}$. La tensione a 360 c/s, resa sinusoidale con un filtro, alimenta, attraverso un amplificatore di potenza, il motore che fa ruotare il cilindro del recristratore. Sia in questo, sia nell'analizzatore, sono stati montati, coassialmente ai motori sincroni, dei motori monofase ad induzione, asincroni, $i$ quali hanno lo scopo di avviare e portare alla velocita di sincronismo i molori sincroni, e di fornire la coppia necessaria per far girare $i$ cilindri. La funzione dei motori sincroni si riduce quindi a quella di ruote foniche regrolatrici della velocità di rivoluzione.

Oltre al reqistratore elettromeccanico, si è costruito un registratore utilizzante un forlio continuo di carta eletrochimica umida.

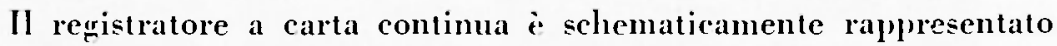
in figr. 7 .

La carta elettrochimica i avolta su un rullo (a) e si svolge lentamente trascinata da un rullo grommato (hisul quale o premuta da un alt ro rullo gommato (c).

Il rullo (a) della carta e racchiuso in un ambiente il più possibile a tenuta per ridure l'evaporazione della carta, la quale deve avere un determinato grado di umidita. La carta esce dall'ambiente suddetto altraverso una fenditura con bordi guamiti di gomma. Questi ultimi esercitano una legrgera azione frenante sulla carta, la quale in tal modo aderisce sulla superficie di un cilindro di cartone bakelizzalo (d), sul quale ì arvolto ad elica 111 filo metallico (e). Nella 
RITRASMESSO mEDANTE Faxsimile DTNAFAX "Ecom Roma,E7-VI-IgSL

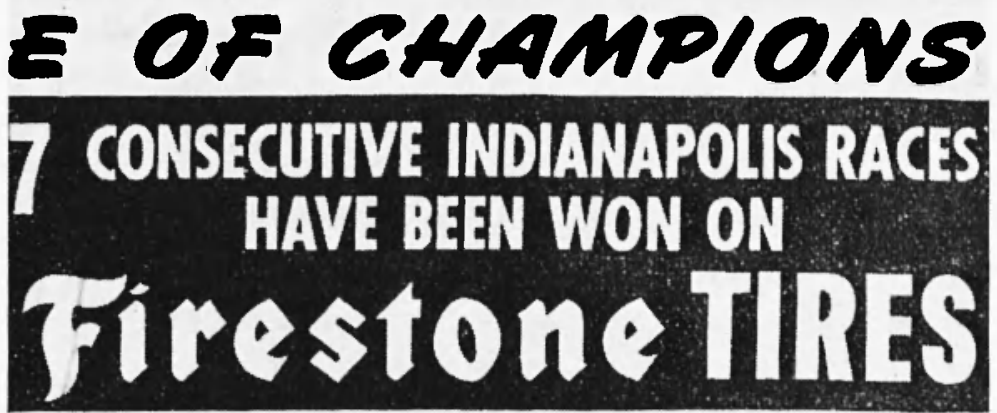

I930-124.002 M.P.H.

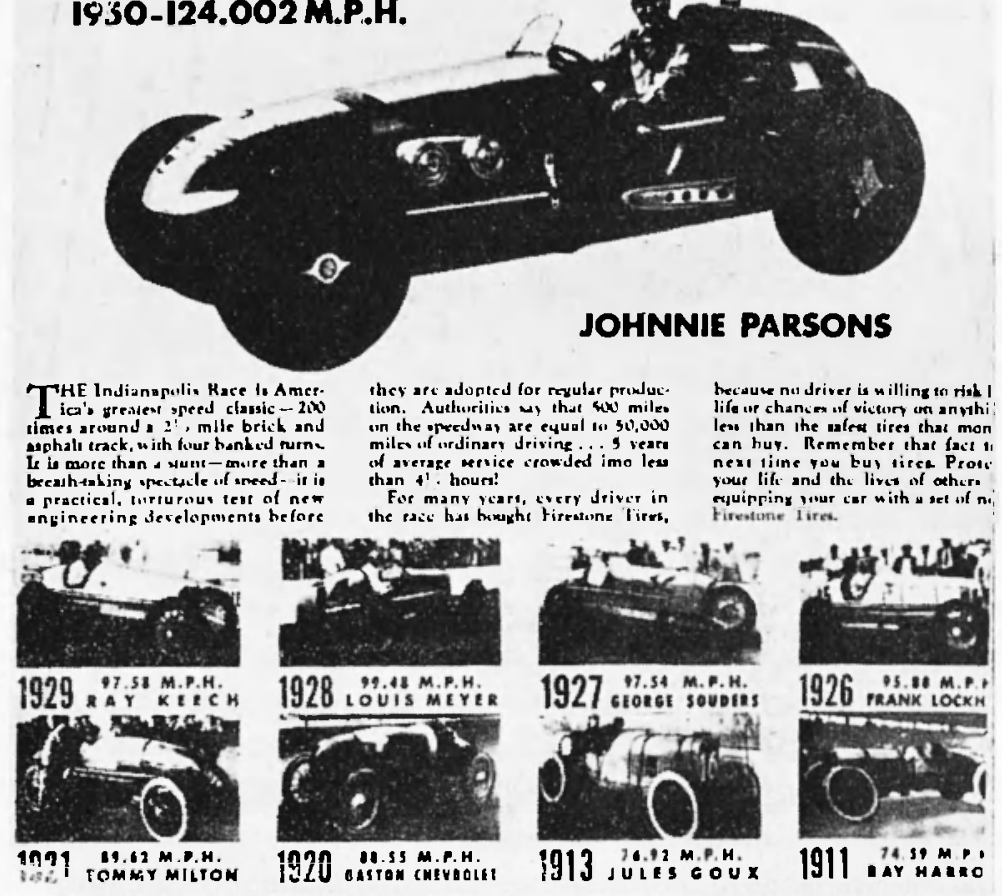

Fig. 13

fig. 7 la carta è stata disegnata trasparente. Sopra la carta è poggiata, di taglio, una barretta ( $f$ ) di ferro (o di altro metallo, a seconda della qualità della carta usata).

Per mezzo del motore sincrono $(g)$ accoppiato ad un motore asincrono il cilindro (d) è fatto ruotare alla medesima velocità di rivolu- 
zione del cilindro sul quale ̀̀ avvolto il documento da trasmettere nell'analizzatore. Il moto del cilindro con l'elica (d) è trasmesso al cilindro d'avanzamento (b) attraverso una serie di ingranaggi ridutlori (h).

Si vede cliaramente che, se il cilindro con l'elica (d) gira nel senso indicato dalla freccia, il punto d'intersezione tra l'elica e la sharretta si sposta con moto uniforme da sinistra a destra, per ritornare "a capo" ad ogni giro del cilindro. Se fosse applicata una differenza di potenziale di $30 \div 40$ volt tra la sbarretta $(+)$ e l'elica $(-)$, la carta che si trova tra l'elica e la sbarretta si annerirebbe lungo una riga trasversale. Dato il moto di trascinamento imposto dal cilindro d'avanzamento (b), questa riga risulta leggemente inclinata rispetto al bordo della sharretta; quando il cilindro con l'elica effettua un giro successivo, viene tracciata un'altra riga parallela alla prima, e cosi via.

Ovviamente tra la sharretta e l'clica non viene applicata una differenza di potenziale continua altro che nel caso in cui si debloa registrare un'immagine completamente nera. In grenerale sarà applicata una differenza di potenziale variabile tra 0 e 35 volt, in funzione della tintegriatura del punto corrispondente esplorato in quel momento dall'analizzatore. Lit larghezza della sharretta corrisponde allo sviluppo del cilindro dell'analizzatore, e l'avanzamento della carta corrisponde all'avanzamento del carrello su cui è montata la lamparla eccitatrice c la fotocellula.

Ove non sia richiesto per ragioni particolari, non è necessario che la registrazione avvenga alla stessa scala del documento originale trasmesso: in generale anzi ̀̀ conveniente una riduzione del formato, poiché nella registrazione le righe sono assai sottili ed apparirebhero separate se la loro distanza fosse maggiore di $1 / 4 \mathrm{di}$ mm. circa, mentre il documento da riprodurre può essere esplorato con una finezza inferiore, al fine di ridurre la banda di frequenza occupata ed il tempo necessario alla trasmissione di un documento di determinata superficie, pur senza ridume sensibilmente la chiarezza.

Il circuito del registratore a carta preparata è rappresentato nella fig. 8.

La sottoportante modulata $\grave{e}$ amplificata in tensione ed in potenza rispettivamente dai tubi $6.1 B+$ e $6.4 Q 5$, e successivamente viene demodulata dal doppio diodo 6.AL5. La tensione (positiva) che si ricava ai capi della resistenza $R$ è sovrapposta alla tensione negativa 


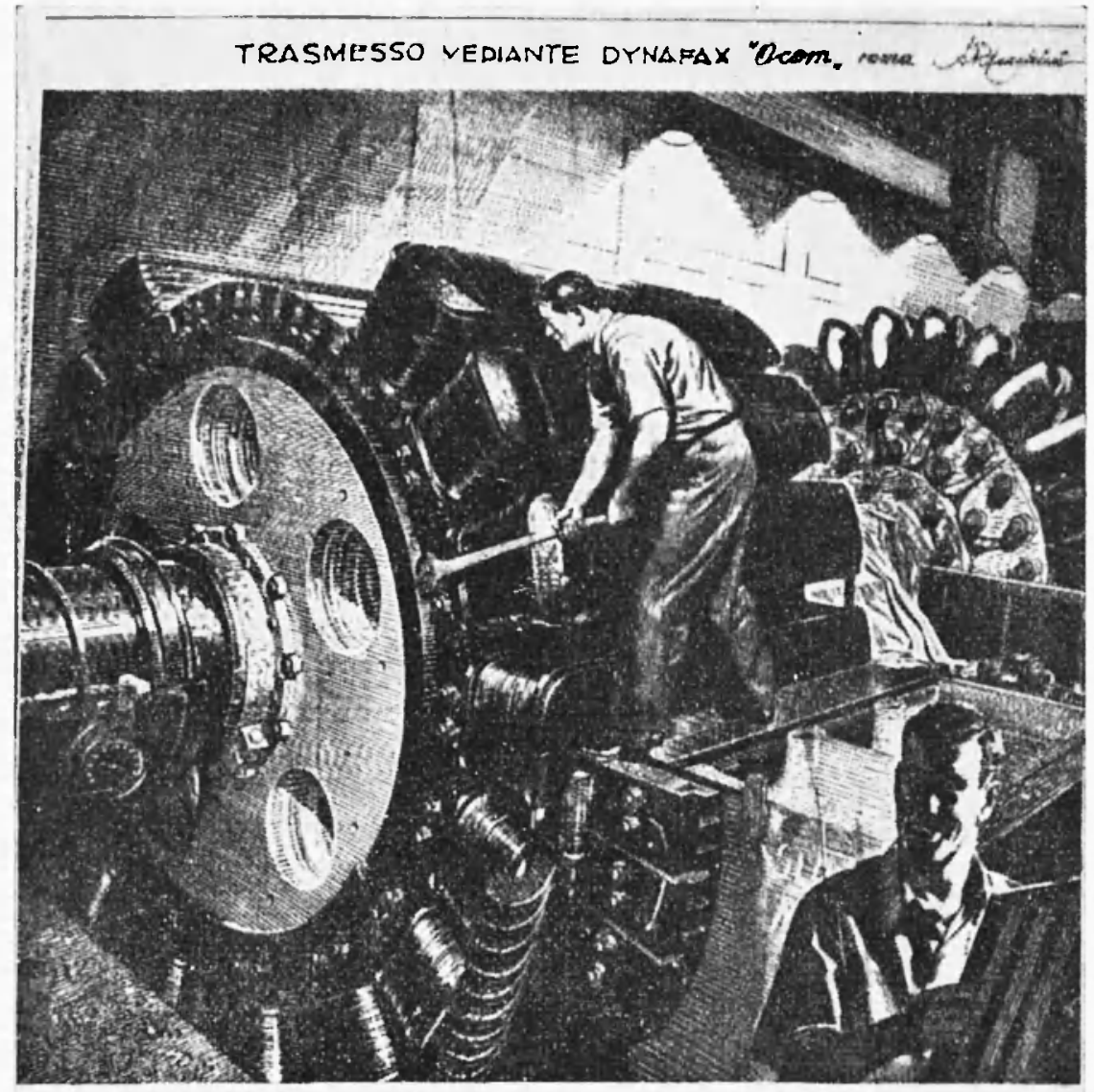

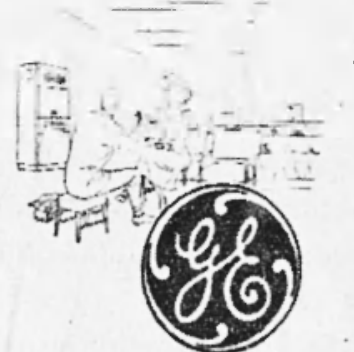

The frindoed of Enowitince

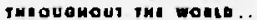

IN INOUSTEY AND MOMI

A flowing streom might delermine

\section{YOUR FUTURR PROOEES}

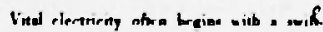

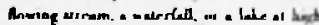
eteration. The fuluer pripotis of gues

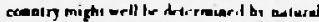

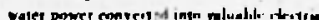

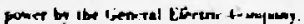

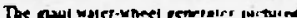

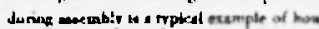

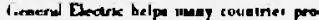

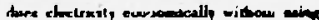

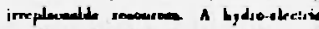

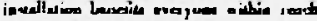

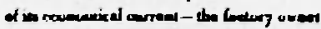

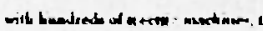

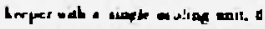

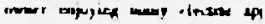

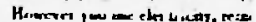

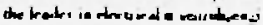

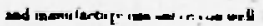
Bactric prothen to for u are avalabte everyot ere. Ieter.

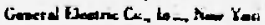

GEMEAL G ELECi ath.

Fig. 14 
di polarizzazione della rriorlia della $6 \mathrm{Y} 6$ connessa a triodo. In tal modo, in assenza di sottoportante, o con il minimo di ampiezza di questa (hianco), la $6 Y 6$ interdetta $e$ non si ha passaggrio di corrente attraverso la carta, che resta bianca. Quando la tensione rettificata, sovrapposta alla tensione di polarizzazione della $6 \mathrm{Y} 6$ sllocca questo tubo, la carta risulta annerita tanto più quanto meno negativa è la griglia.

La resistenza della carta umida, tra la spirale e la larretta, è dell'ordine di un miqliaio di olım, e l'annerimento completo si ha quando la carta d attraversata da una carica di circa $100 \mu$ Couloml.

Considerazioni generali sui sistemi di sincrontsmo. - Le apparecchiature descritte, costruite in piccola serie dalla ditta Ocem di Roma $\left({ }^{1}\right)$, sono state terminate (ad esclusione del regristratore a carta elettrochimica continua, piì recente) nel gennaio del 1951.

Da allora, con le suddette apparechiature, sono state fatte molte prove di trasmissione via filo e via radio in $J^{\prime} H F$ ed $H F$. Inoltre sono state costruite altre apparecchiature utilizzanti la stessa rete di distriluzione dell'energia elettrica per il sincronismo, con partenza ed arresto completamente automatici, ed è stato sviluppato uno studio accurato per la costruzione di diapason himetallici.

In lase a questo complesso di esperienze lo scrivente è giunto alla conclusione che, quando si debha stalilire una comunicazione tra due punti allhastanza ben collegati (per es. via cavo o via radio $l H F$ ), come accade nella maggior parte dei casi pratici, e le reti di alimentazione siano a frequenze differenti, il sistema di sincronismo con utilizzazione della sottoportante possa trovare applicazioni veramente interessanti.

Del resto questo sistema ricorda molto i più recenti sistemi di sincronismo impiegati nella Televisione ed inoltre si può ritenere che delle apparechiature hasate su questo sistema, qualora potessero essere studiate per una effettiva produzione in serie, sarehbero sensil,ilmente più economiche di quelle con sincronismo a diapason. La fig. 9 mostra una registrazione ottenuta in condizioni particolarmente sfavorevoli: la trasmissione è statal effettuata su onda di 60 metri circa: l'interferenza è stata prodotta da un impianto a raggi $\mathrm{X}$ del tipo "Trabacchi-Corlino" funzionante in un locale attiguo a quello in cui si trovava il ricevitore: il rapporto segnala disturbo era inferiore a -20

(1) di Astegiano Mario \& C. 


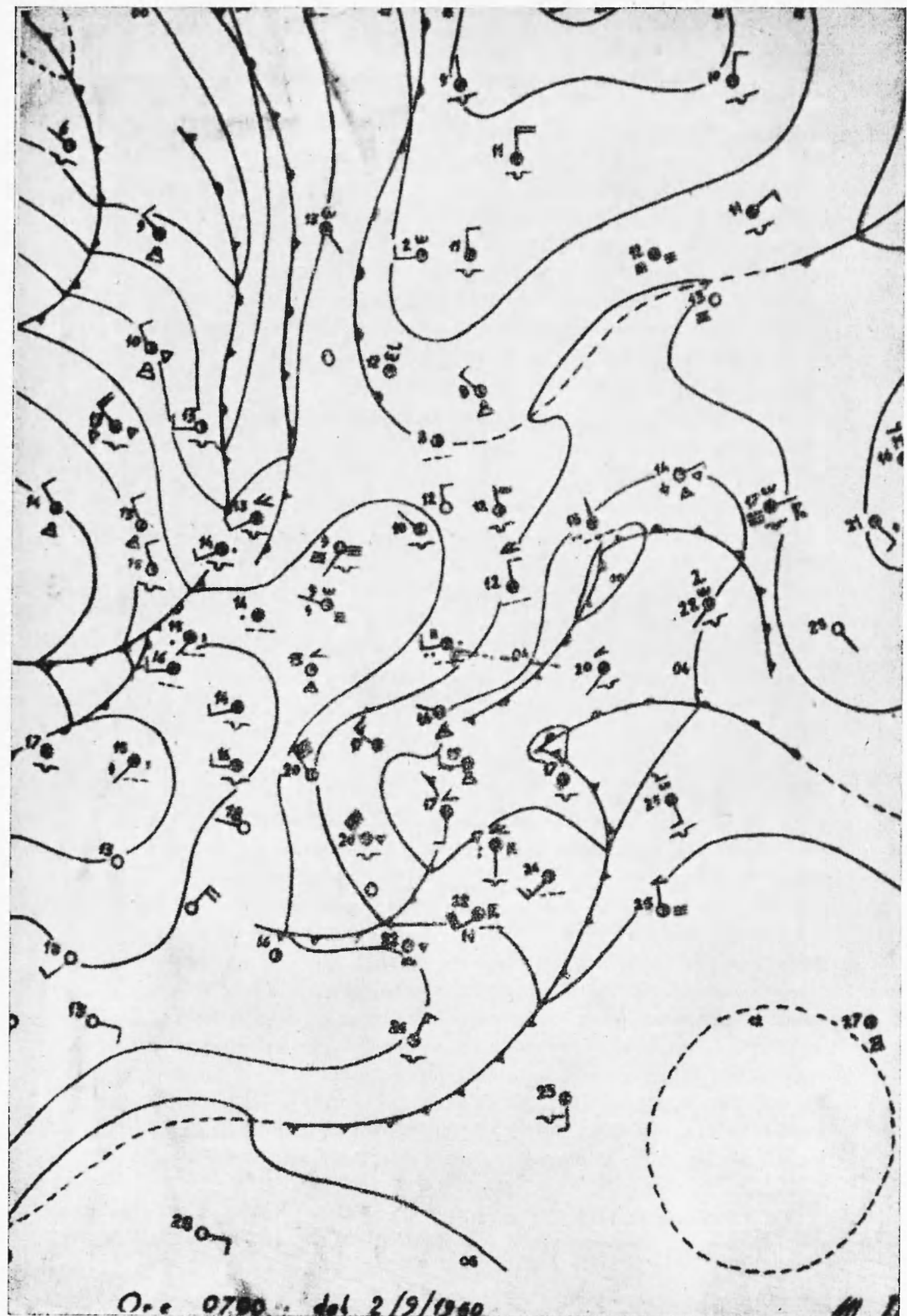

Fig. 15 
Al birca, ciò nonostante il sincronismo si è mantenuto. Il registratore era del tipo elettromeccanico.

Per contro, il sistema attuale ha a suo svantaggio l'inconveniente di non poter essere adoperato in trasmissioni con Frequency Slift, in quanto queste ultime apparecchiature non si prestano ad utilizzazioni di tipo telefonico.

Qualora sia possibile alimentare le apparecchiature fac-simile mediante reti di distribuzione dell'energia elettrica aventi la medesima frequenza, ¿̀ ovvio che l'utilizzazione della rete stessa per il sincronismo costituisce il sistema più logico, sicuro ed economico: dato che in un tempo più o meno prossimo si dovrà arrivare alla sincronizzazione di tutte le reti nazionali, questo sistema di sincronismo dovrà costituire il sistema dell'avvenire nelle comunicazioni tra punti fissi; non potrà però ovviamente essere utilizzato per apparecchiature mobili.

Un'ottima soluzione del problema sia dal punto di vista tecnico. sia dal punto di vista commerciale, consiste senza dubbio nella costruzione di apparati adatti al funzionamento con sincronismo mediante la rete, utilizzando piccoli motori asincroni sincronizzati, e nella costruzione di unità separate costituite da un oscillatore a frequenza stabilizzata a diapason, una sequenza di divisori per ottenere una frequenza adatta al funzionamento di tali motori (50 o 60 c/s) - è da notare che diapason a frequenza molto bassa non garantiscono sufficiente stabilita - e da un amplificatore di potenza adatto all'alimentazione del motore. Tali unità saranno impiegrate in ognni occasione in cui nei due punti tra $i$ quali si deve effettuare la trasmissione di immagini non si dispone della stessa rete o di reti sincronizzate.

In un prossimo articolo ci si ripromette la descrizione di un'apparecchiatura costruita secondo tale criterio.

La fig. 10 i la fotografia dell'analizzatore. La parte posteriore del mobile porta due hordi con fori filettati, in modo da costituire un telaio normalizzato. La parte elettronica che vi ì allogata, occupa solo la parte superiore del telaio, ed è alta $31 \mathrm{~cm}$.

Inferiormente possono trovar posto altre apparecchiature, quali radiotrasmettitori per ponti radio, amplificatori, modulatori ecc.

La fig. 11 ì la fotografia del registratore. Il mobile è costruito con lo stesso criterio del mobile dell'analizzatore. Lo chassis del registratore ha le stesse dimensioni dello chassis dell'analizzatore. 
La fig. 12 rappresenta un tipo di registratore a carta continua, completamente automatico, di dimensioni ridotte.

Le altre fotografie rappresentano dei campioni di immarini trasmesse (via cavo telefonico): la riproduzione in fig. 13 ha dimensieni

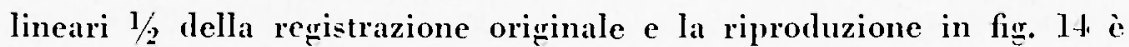
circa $2 / 3$ dell'origrinale. La figr. 15 ha la stessa scala della regristrazione origrinale: la cartina meteorologrica trasmessa era disegnata su carta lucila.

Diserni, schemi e fotografie sono stati cortesemente forniti dalla ditta OCEM.

Tengo molto a ringraziare l'Ing. Fnzo Cambi per tutti i preziosi consigli e per le numerose discussioni chiarificatrici.

\section{RIASSUNTO}

Sono descritti alcuni perfezionamenti apportati ad un apparato per le trasmissioni di immagini. I perfezionamenti riguardano particolarmente il sistema di sincronismo tra analizzatore e registratore. Si aggiungono alcune considerazioni relative alla scelta di un sistema di sincronismo.

\section{SUMIIRY}

Some refinements pertaining to an apparatus for the transmission of images are described. These refinements apply particularly to a method of synchronizing the analyzor and detector. Various considerations have been added concerning the choice of a synchronous system. 